\title{
Progresividad del gasto público en salud en México: 1984-2002
}

\section{Progressivity of public expenditure on health in Mexico: 1984-2002}

\author{
César Octavio Vargas-Téllez*
}

\begin{abstract}
This study attempts to calculate the progressivity of health expenditure by institution, type of medical care and its behavior for 16 years. Using analysis of incidence and micro data, we generated distributions of transfers of public expenditure on health per family. The analysis is completed with the calculation of inequality indexes; the results show a gradual improvement in the progressivity of health expenditure in the study period, with higher progressivity of health services provided by the Secretariat of Health and fewer improvements in ISSSTE and Pemex services. By type of medical care, preventive services seem more progressive than curative and hospital services. The results support the hypothesis that universal health care and their unification through a single entity would substantially improve the fairness of the public health system in Mexico.
\end{abstract}

Keywords: Mexico, public expenditure, health care, progressivity, distribution.

\section{Resumen}

En este trabajo intentamos calcular la progresividad que tiene el gasto en salud por institución, tipo de cuidados médicos y su evolución a lo largo de 16 años. Utilizando análisis de incidencia y microdatos, se generaron distribuciones de las transferencias del gasto público en salud por familia. El análisis se completa con el cálculo de índices de desigualdad, los resultados arrojan una paulatina mejoría en la progresividad del gasto en salud en el tiempo de estudio, con mayor progresividad de los servicios de salud prestados por la Secretaría de Salud y menores avances en los servicios del Issste y Pemex. Por tipo de cuidados médicos, los servicios preventivos se han desarrollado más que los servicios hospitalarios y curativos. Los resultados sostienen la hipótesis de que la universalización de los servicios de salud y su unificación en una sola entidad proveedora mejoraría sustancialmente la equidad del sistema de salud pública en México.

Palabras clave: México, gasto, salud, progresividad, distribución.

* Universidad Autónoma Metropolitana, Cuajimalpa, México. Correos-e: cvargas@correo.cua. uam.mx, covargastellez@hotmail.com. 


\section{Introducción}

Durante el cambio estructural que presentó la economía mexicana en los años ochenta y noventa, se agudizaron las desigualdades sociales, afectadas por un ajuste del gasto social como consecuencia de una reformulación del papel del Estado en la economía. Es en este contexto, donde cada vez tiene más sentido preguntarse por la eficiencia de las políticas sociales y sus efectos redistributivos; y el gasto en salud no puede ser ajeno a esta revisión de las políticas públicas. Si se parte del hecho de que la salud es un bien privado, pero es deseable que se provea como bien público, ${ }^{1}$ dados los efectos que tiene en el bienestar social de la población, es pertinente conocer qué tan efectivo y equitativo ha sido el gasto público en salud.

México presenta indicadores de salud (mortalidad y desnutrición) similares a países con un nivel del PIB per cápita muy por debajo del mexicano. ${ }^{2}$ Por ello es necesario saber qué ha pasado con las políticas de salud en los últimos ańos y, en especial, con la eficiencia del gasto, para entender la situación actual. Aunque se han hecho esfuerzos encaminados a mejorar el perfil de salud de la población mexicana, como el Seguro Popular, se debe entender en qué contexto se están implantando los nuevos cambios en materia de salud pública, ya que ello condicionará en buena medida sus alcances.

Debido a lo anterior, cabe reflexionar hasta qué punto un sistema de universalización de los servicios de salud son eficientes y equitativos. Para ello, en este trabajo se hace una breve revisión de la evolución de la progresividad en el gasto de las instituciones públicas proveedoras de servicios de salud, durante un periodo de 18 años (1984-2002). Cabe hacer notar que este lapso contempla el análisis de cuatro versiones de la Encuesta Nacional de Ingreso y Gasto de los Hogares (ENIGH), ${ }^{3}$ que son la base de microdatos, y de la Encuesta Nacional de Salud de 2000 (Ensa-2000). El periodo se justifica porque abarca de manera adecuada los cambios en las políticas públicas que se han venido gestando en México, a raíz del cambio de modelo de desarrollo elegido.

\footnotetext{
${ }^{1}$ Desde el punto de vista estrictamente económico, la salud es un bien cuya exclusión puede ser barata y fácil, simplemente con aplicar cuotas. Sin embargo, aunque sea fácil no es deseable dadas sus externalidades positivas, por lo que se convierte en un bien público.

${ }^{2}$ De acuerdo con Scott (2000), Sri Lanka, con un ingreso per cápita cinco veces menor, presentaba mejores niveles en mortalidad infantil.

${ }^{3}$ Es importante decir que las ENIGH se levantan cada dos o tres años, de tal suerte que en el tiempo en que se hizo el presente estudio, la última, de 2008, aún no había sido publicada.
} 


\section{Cuadro 1}

\section{Gasto público programable ejercido en el subsector salud (millones de pesos constantes de 2002)}

\begin{tabular}{rrrrrcc}
\hline Año & Total & SSA $^{b}$ & ISSSTE & IMSS $^{c}$ & $\begin{array}{c}\text { Organismos, empresas, } \\
\text { fondos y fideicomisos, } \\
\text { subsidios }\end{array}$ & $\begin{array}{c}\text { Gasto } \\
\text { per cápita }\end{array}$ \\
\hline 1983 & 107,719 & 10,163 & 25,428 & 69,195 & 2,932 & 1,473 \\
1984 & 103,032 & 10,976 & 21,131 & 66,611 & 4,315 & 1,375 \\
1985 & 104,047 & 11,334 & 22,328 & 65,568 & 4,817 & 1,355 \\
1986 & 110,816 & 10,920 & 20,818 & 72,429 & 6,649 & 1,413 \\
1987 & 101,370 & 10,938 & 22,370 & 62,907 & 5,156 & 1,266 \\
1988 & 104,629 & 11,121 & 20,795 & 64,877 & 7,836 & 1,279 \\
1989 & 112,519 & 10,673 & 20,840 & 73,058 & 7,949 & 1,346 \\
$1990^{\mathrm{a}}$ & 90,433 & 16,261 & 6,681 & 67,491 & $\mathrm{n} . \mathrm{d}$. & 1,059 \\
1991 & 109,563 & 19,411 & 12,805 & 77,347 & $\mathrm{n} . \mathrm{d}$. & 1,258 \\
1992 & 120,718 & 21,362 & 13,122 & 86,235 & $\mathrm{n} . \mathrm{d}$. & 1,360 \\
1993 & 129,848 & 23,232 & 9,443 & 94,287 & 2,885 & 1,436 \\
1994 & 144,754 & 25,472 & 14,043 & 101,873 & 3,365 & 1,573 \\
1995 & 119,251 & 23,072 & 8,077 & 84,672 & 3,430 & 1,274 \\
1996 & 114,751 & 24,671 & 8,734 & 77,651 & 3,695 & 1,207 \\
1997 & 131,624 & 33,679 & 10,410 & 83,358 & 4,177 & 1,363 \\
1998 & 142,817 & 37,700 & 11,343 & 89,406 & 4,369 & 1,459 \\
1999 & 163,171 & 47,371 & 12,626 & 98,693 & 4,482 & 1,644 \\
2000 & 165,723 & 51,297 & 13,966 & 95,568 & 4,893 & 1,648 \\
2001 & 175,800 & 55,090 & 15,006 & 100,301 & 5,403 & 1,726 \\
2002 & 180,847 & 57,599 & 15,101 & 102,475 & 5,672 & 1,755 \\
\hline
\end{tabular}

a A partir de este año, la serie se ha completado con datos proporcionados directamente por la ssA.

${ }^{\mathrm{b}}$ A partir de 1999 se agregan las cantidades de gasto de los estados.

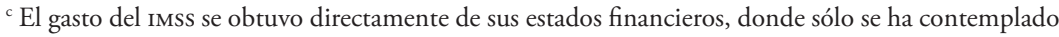
el gasto exclusivo en salud y se excluye el gasto en pensiones.

d A partir de 1993 la columna sólo representa datos de PEMEx, ya que otros organismos de salud, fondos y fideicomisos están representados en la columna de Gobierno Federal (ssa).

Fuente: Elaboración propia con datos de la SSA, IMSS, ISSSTE e informes presidenciales.

\section{Evolución y distribución del gasto en salud}

Para obtener un crecimiento económico socialmente sustentable, mejorar de manera equitativa la distribución de los recursos y construir un capital humano adecuado, es menester tener máximos niveles de satisfacción en salud, sostenidos con niveles adecuados de gasto público.

Mediante la observación del comportamiento del gasto público en salud (cuadro 1), se aprecia que no siempre se sostuvo el nivel de gasto, por el contrario, hubo un deterioro constante durante toda la década de los ochenta, que lo llevaron a su nivel más bajo en 1990, dentro del periodo de estudio.

Durante los casi 20 años que contempla el periodo de estudio, la población mexicana aumentó en más de 25 millones, que aunado a la errática y pobre trayectoria del gasto en salud, dieron como resultado la 
acumulación de rezagos en materia de salud pública. Ello se confirma en el hecho de que, si bien aumentó el gasto en salud en $67.9 \%$ en términos reales en el periodo 1983-2002, el gasto per cápita sólo lo hizo en $19.10 \%$ (cuadro 1).

Por si los anteriores factores no fuesen suficientes en el deterioro del sistema de salud, éste registró una importante inequidad en cuanto a cobertura, debido a la falta de un sistema universal, lo que propició que una parte sustancial de la población careciera del servicio.

A manera de ejemplo, en 2002 apenas 54\% de la población tenía acceso a atención médica a través del sistema público de seguridad social, ofrecido por las dos principales instituciones proveedoras: el Instituto Mexicano del Seguro Social (IMss) y el Instituto de Seguridad y Servicios Sociales de los Trabajadores del Estado (ISSSTE), las cuales absorbían 65\% del gasto total destinado a salud.

Por otro lado, entre 35 y 40\% de la población no contaba con algún tipo de cobertura sanitaria (pública o privada), que en principio debía ser atendida por la Secretaría de Salud, mediante su red nacional de hospitales generales y clínicas rurales, cuyo presupuesto es la mitad del que emplean las otras instituciones proveedoras de salud. De esta manera, el gasto per cápita destinado a salud para la población asegurada en el año 2002, fue 2.3 veces superior al asignado para la población no asegurada. Si bien esta relación cobertura-gasto ha mejorado en los últimos años, aún dista de ser igualitaria.

Una razón de ello es la fragmentación del sistema de salud pública, el cual se compone de dos grandes subsectores: el primero incluye organizaciones públicas de seguridad social, entre los que se encuentran el IMSs (el principal proveedor del sistema de seguridad social que atiende sobre todo a trabajadores del sector privado ${ }^{4}$ y autónomos de la economía formal), el Issste (básicamente empleados de la administración pública federal y locales) y sistemas más pequeños adscritos a ciertas industrias, como Petróleos Mexicanos (Pemex) o a secretarías como las de Defensa y Marina, donde por el perfil de los afiliados tradicionalmente han sido más beneficiados en los estamentos urbanos. A esta población asegurada pertenecen los trabajadores más favorecidos, puesto que son los que están integrados en la economía formal, y aunque pagan cuotas por acceder al servicio, el gasto público destinado a estas instituciones es mucho mayor que los ingresos obtenidos por cuotas.

El segundo gran grupo es el que encabeza la Secretaría de Salud con su red de hospitales rurales, generales y de especialidades, los cuales atien-

${ }^{4}$ Existen algunos colectivos de trabajadores cubiertos, que laboran para entidades públicas, sobre todo empresas paraestatales (públicas). 


\section{Cuadro 2}

Estructura del gasto programable ejercido en el subsector salud

\begin{tabular}{lrrrc}
\hline Año & SSA & ISSSTE & IMSS $^{c}$ & $\begin{array}{c}\text { Organismos, empresas, } \\
\text { fondos } y \text { fideicomisos, }^{\text {subsidios }^{d}}\end{array}$ \\
\hline 1983 & & & 2.7 \\
1984 & 9.4 & 23.6 & 64.2 & 4.2 \\
1985 & 10.7 & 20.5 & 64.7 & 4.6 \\
1986 & 10.9 & 21.5 & 63 & 6 \\
1987 & 9.9 & 18.8 & 65.4 & 5.1 \\
1988 & 10.8 & 22.1 & 62.1 & 7.5 \\
1989 & 10.6 & 19.9 & 62 & 7.1 \\
$1990^{\mathrm{a}}$ & 9.5 & 18.5 & 64.9 & $\mathrm{n} . \mathrm{d}$ \\
1991 & 18 & 7.4 & 74.6 & $\mathrm{n} . \mathrm{d}$ \\
1992 & 17.7 & 11.7 & 70.6 & 2.2 \\
1993 & 17.7 & 10.9 & 71.4 & 2.3 \\
1994 & 17.9 & 7.3 & 72.6 & 2.9 \\
1995 & 17.6 & 9.7 & 70.4 & 3.2 \\
1996 & 19.3 & 6.8 & 71 & 3.2 \\
1997 & 21.5 & 7.6 & 67.7 & 3.1 \\
1998 & 25.6 & 7.9 & 63.3 & 2.7 \\
1999 & 26.4 & 7.9 & 62.6 & 3 \\
2000 & 29 & 7.7 & 60.5 & 3.1 \\
2001 & 31 & 8.4 & 57.7 & 3.1 \\
2002 & 31.3 & 8.5 & 57.7 & \\
\hline
\end{tabular}

Fuente y notas: Igual que el cuadro 1.

den a una población abierta que no tiene una cobertura social. En esta categoría se encuentra el sistema IMss-Oportunidades, financiado totalmente con fondos públicos y administrado por el Imss. Cabe mencionar que el perfil mayoritario de esta población es rural o urbano marginal.

Esta fragmentación implica una alta desigualdad en la distribución del gasto. Analizando la estructura del gasto público en salud, se observa que el IMSS y el ISSSTE tradicionalmente han acaparado la mayor parte del presupuesto del sector, aunque cubran una población menor que la red de hospitales de la Secretaria de Salud (cuadro 2).

Tal desigualdad se proyecta a varios estratos, de modo que existe una desproporción entre estados pobres y ricos, entre ámbitos rurales y urbanos y entre población asegurada y no asegurada. Es ilustrativo mencionar que mientras los asegurados por Pemex reciben recursos anuales per cápita por 5,000 pesos (el promedio nacional era de 1,750 pesos en el año 2002), la población no asegurada -también llamada población abiertasólo recibe alrededor de 200 pesos. Es evidente que la dualidad del sistema de salud, con su duplicidad de funciones, mantiene la desigualdad e ineficiencia en el acceso y financiamiento de los recursos. 


\subsection{Financiamiento del gasto}

Para completar la breve revisión del sistema de salud pública y su análisis de incidencia, se exponen las diferencias de financiamiento de los dos subsistemas que lo componen.

El sistema de salud está financiado en su mayoría por impuestos sobre nómina y subsidios del gobierno federal. Examinando cada subsistema proveedor de salud (IMSS, ISsSTE y Pemex), se encuentra que son financiados tripartitamente por contribuciones obligatorias de sus afiliados (trabajadores), empleadores y gobierno.

Sin embargo, fuentes del IMss han detectado una importante evasión en dichas contribuciones. Por ejemplo, mientras en 1993 el 20\%5 de los trabajadores fueron registrados con ingresos de un salario mínimo, en contraste, según la Encuesta Nacional de Empleo, sólo 2.5\% registraba esta categoría salarial. Para corregir dicha situación, se ha tratado de ampliar la cobertura que ofrece el IMSs mediante rebajas en las cuotas que aportan los trabajadores y sus empleadores, con el fin de reducir costos laborales y atraer más población al sistema formal de seguridad social y evitar el fraude existente. De esta manera, en 1997 se implementó una reforma del Imss que contempla una cuota fija a los empleadores, dentro de una parte de las contribuciones, con el objetivo de evitar una total vinculación con el nivel de ingreso.

Con estas medidas se espera que con la combinación de menores impuestos sobre el ingreso y un aumento en el financiamiento del sistema a través de un nivel de tributación general, se atraiga a una importante cantidad de trabajadores a la economía formal, pretendiendo disminuir la alta evasión en las contribuciones. Esto se circunscribe con el argumento de que la financiación de un sistema de salud a través de contribuciones (sobre nóminas), proporcionales o progresivas, convierten los pagos a la seguridad social más en un impuesto que una prima de seguro, incidiendo negativamente en la afiliación del sistema de salud. Por tanto, un subsidio a este esquema se puede justificar por las externalidades positivas que origina (OECD, 1997), aunque minimizadas por el actual sistema dual de procuración de salud, el cual deja a una gran parte de la población sin cobertura.

Por su parte, la otra gran institución proveedora de salud y seguridad social, el IssSTE, está casi totalmente financiada por el Estado, ya que los asegurados son trabajadores públicos y su empleador es el Estado, y por tanto éste paga las cuotas patronales, más las transferencias directas utili-

${ }^{5}$ De acuerdo con la Memoria Estadística, año 2000, capítulo xıv, salario base de cotización. 
zadas para sostener a la institución, de manera que sólo una mínima parte consta de contribuciones salariales de los empleados.

En lo referente al financiamiento de los servicios médicos de la ssa que se dirigen a la población abierta, básicamente estos los sostiene el gobierno federal mediante el sistema tributario general. También existe una pequeña fracción financiada por gobiernos estatales y municipales, que en muchos casos son transferencias federales. Recientemente se ha intentado aumentar la generación de recursos propios a través de cuotas a los usuarios del servicio, las cuales son proporcionales a su nivel de ingreso, y que alcanzaron $10 \%$ de los ingresos totales a principios de la década actual.

Una vez revisados brevemente los diferentes subsectores de salud, se llega a la conclusión de que México tiene un sistema de salud financiado mayoritariamente por el Estado.

\section{Cálculo de la incidencia}

Imputar el gasto en salud que destina el Estado, siempre es un ejercicio complejo y no exento de polémica, porque incluye los costos administrativos, muchas veces exorbitantemente altos y que además no inciden de manera directa en el beneficio real de las familias. Si a esto se ańade la fragmentación del sector, donde cada institución tiene una estructura de costos y población beneficiada muy diferentes, el ejercicio de incidencia se complica un poco más.

En este caso, el ejercicio de incidencia se apoya en dos bases de datos: la Encuesta de Ingreso y Gasto de los Hogares (ENIGH) para cada año de referencia, y la Encuesta Nacional de Salud (Ensa-2000), para después sumar el gasto en salud obtenido a través de estas dos encuestas y contrastarla con las cuentas nacionales.

Para obtener las transferencias en salud hacia las familias, en un primer intento se utilizaron los ingresos no monetarios reportados en las ENIGH, lo que significa sólo los pagos y regalos en especie hacia los hogares, sin embargo los resultados fueron poco realistas, ya que no existe en dichas encuestas información directa sobre los montos de las transferencias en salud y beneficiarios del sistema. Básicamente se contabilizó el concepto de bienes y salud adquiridos en instituciones públicas (pagos y regalos en especie), asumiendo la cantidad reportada por las familias como el monto de las trasferencias públicas en salud. Sin embargo, al agregar estas cantidades y contrastarlas con los agregados del gasto en salud por 
institución, ${ }^{6}$ se encuentra un subreporte excesivo, ${ }^{7}$ ya que sólo se reporta $14.3 \%$ del gasto hecho por el Issste, $10 \%$ del IMss y $4 \%$ de la Secretaría de Salud.

En un segundo intento, para solucionar este problema se tomaron en cuenta las variables: prestaciones sociales y servicios médicos reportadas en las ENIGH, para identificar el tipo de institución proveedora de salud por familia, ${ }^{8}$ en los casos donde no la había, éstos se imputaban a los servicios del la Secretaría de Salud (SA), siempre que no declarasen que usaban servicios médicos privados. Posteriormente, con base en información de la Ensa-2000, se construyeron precios sombra del costo de provisión de los servicios de salud por institución, como variable proxy del monto de trasferencias por individuo y por familia.

Para la construcción de dichos precios, se tomaron como base los gastos promedio de bolsillo que hizo cada familia en su última visita al médico, por tipo de institución médica y por tipo de servicio. Al respecto, y de acuerdo con la Ensa, existen tres tipos de servicios médicos: preventivos, curativos y hospitalarios. A su vez, los gastos en servicios preventivos y curativos se componen de: costo de la consulta, medicamentos y análisis de laboratorio; mientras que los hospitalarios incluyen: gastos por hospitalización y otros gastos de hospitalización. Así, una vez sumados estos componentes de los tres servicios médicos, se obtuvo el total para cada tipo.

Dado que el gasto promedio reportado de servicios médicos es familiar, para obtener el gasto promedio por persona, simplemente se tomó en cuenta el número promedio de personas que hicieron uso de estos servicios por familia, ${ }^{9}$ lo cual es de gran utilidad ya que, posteriormente, al combinar la tasa de probabilidad de asistencia al médico por sexo y edad, inferida de la Ensa-2000, se puede imputar el monto de transferencia médica por individuo, propiciando mayor exactitud en la estimación en el uso de los servicios de salud, ya que ello permite calcular las diferentes probabilidades de utilizar servicios médicos por edad y sexo, como sucede en la vida real.

Puesto que la información surgió de la Ensa-2000, sólo se había obtenido el gasto de bolsillo por persona por tipo de institución para el año $2000,{ }^{10}$ sin embargo se necesitaba deducir el de los años 1984, 1989, 1996

\footnotetext{
${ }^{6}$ SSA, IMSS, ISSSTe, Pemex, Sedena y Secmar.

${ }^{7}$ Estas cantidades se toman de la ENIGH 2002 y son similares a las encontradas por Scott (2004).

${ }^{8}$ En los casos donde existían dos o más instituciones proveedoras de salud en una familia, se tomó la del cabeza de familia.

${ }^{9}$ Es importante señalar que el número promedio de personas por hogar que hacen uso de los servicios médicos varía por tipo de servicio.

${ }^{10}$ No existen otras encuestas de salud para otros años, al menos en los términos y resultados de la del año 2000.
} 
y 2002. Con el fin de obtener el gasto en salud para esos años, se hizo el supuesto de que el gasto sería el mismo que en el año 2000, de manera que simplemente utilizando el deflactor implícito de precios para el sector salud se obtuvo el gasto en salud de bolsillo por persona en precios corrientes, compatible con la información que reporta cada ENIGH. Volviendo al supuesto de que la estructura del gasto de bolsillo se mantiene constante para todos estos años; en principio podría parecer un supuesto poco realista, sin embargo, cuando se analiza que el comportamiento del gasto en salud reportado por la ENIGH se basa en la probabilidad de asistir al médico por parte de las familias y/o individuos, y se establece que dicha probabilidad varía poco en el tiempo, ya que la probabilidad de asistir al médico es una variable relativamente estática, entonces el supuesto cobra credibilidad. A pesar de ello, hay que reconocer que la polémica sobre el supuesto puede tener más fundamento para los años 1984 y 1989, ya que para 1996 y 2002 realmente existe poca distancia cronológica al año 2000 tomado como base, y por tanto el cambio en los patrones de comportamiento de asistencia al médico de la gente (factor que podría afectar la probabilidad de utilizar servicios médicos de manera importante), es mínimo en el corto plazo. Una vez obtenido el gasto de bolsillo por persona y por institución, se construye la variable proxy, que podría indicar la cantidad subvencionada o transferida por parte del Estado a la población. Así, tomando como base el gasto privado de bolsillo por persona, se obtendrá el subsidio para cada tipo de servicio médico, simplemente restando el gasto de bolsillo devengado por cada institución pública al gasto privado. Entendiéndose a la diferencia resultante, como el subsidio en salud por parte de las instituciones proveedores del servicio, dado que se asume que el gasto privado está determinado por los precios dictados por el mercado (cuadro 3).

El siguiente paso es cargar estas trasferencias en salud a las familias, para ello se buscaron los factores que más incidencia tienen en el uso de los servicios médicos por su tipo, debido a que sería poco acertado cargar por igual los subsidios a toda la población. De acuerdo con la Ensa-2000, factores como el tamaño del lugar de residencia de la población o el nivel de ingreso económico, tienen un efecto mínimo en la tasa de demanda de servicios médicos. Otros, como la entidad federativa donde reside el paciente, registraron pocas diferencias entre sí, en cambio, donde comienzan a existir diferencias en la tasa de asistencia, es el estatus de afiliación a alguna institución pública proveedora de servicios médicos. Mientras que la edad y el sexo fueron los factores más importantes para determinar la tasa de utilización de los servicios médicos, de esta manera, utilizando las tasas de asistencia por tipo de servicio médico, se cargó la transferencia o subsidio en el servicio de salud, ponderada por la probabilidad o tasa 


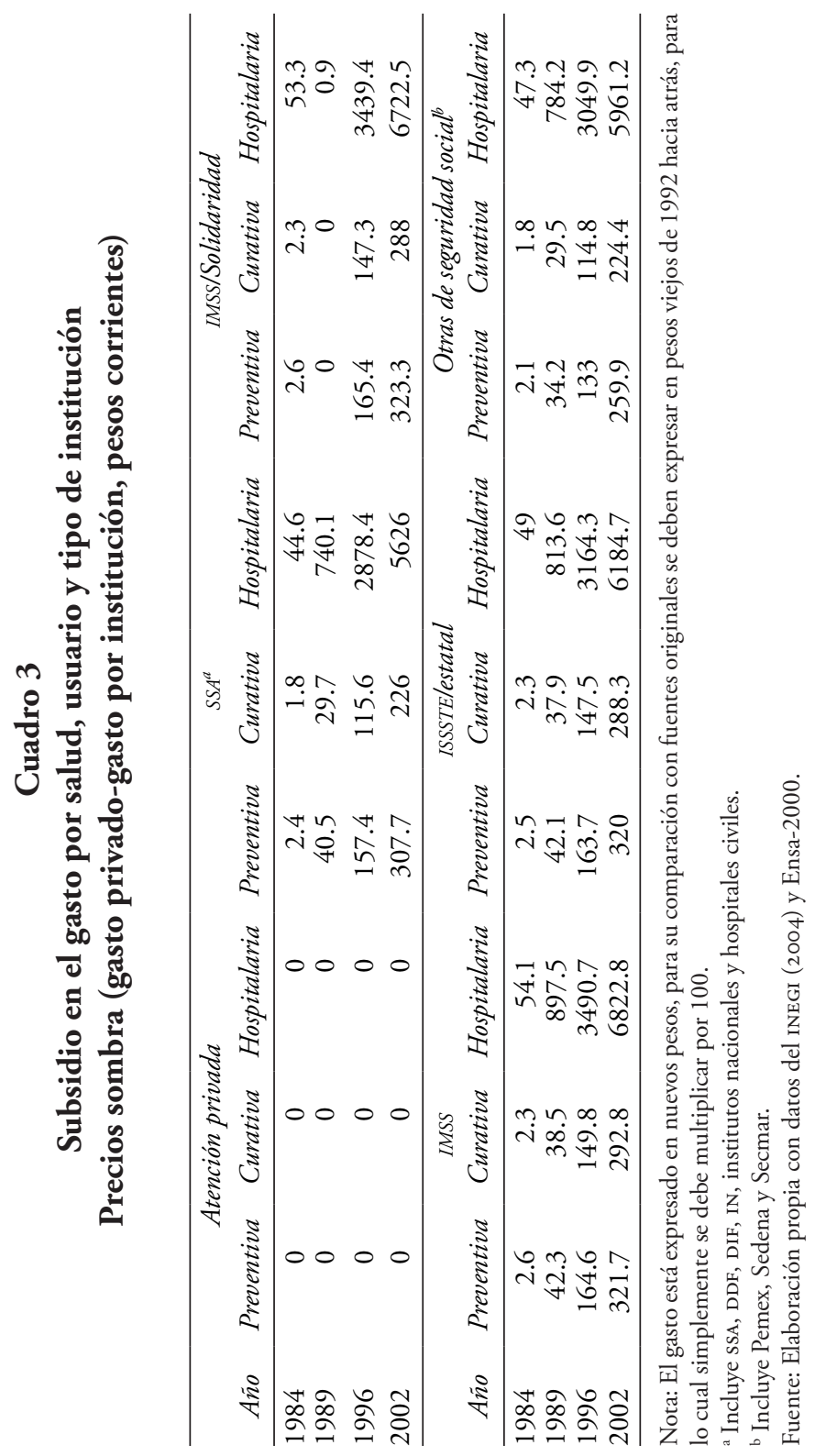




\section{Cuadro 4 \\ Tasa de uso por tipo de servicio de salud}

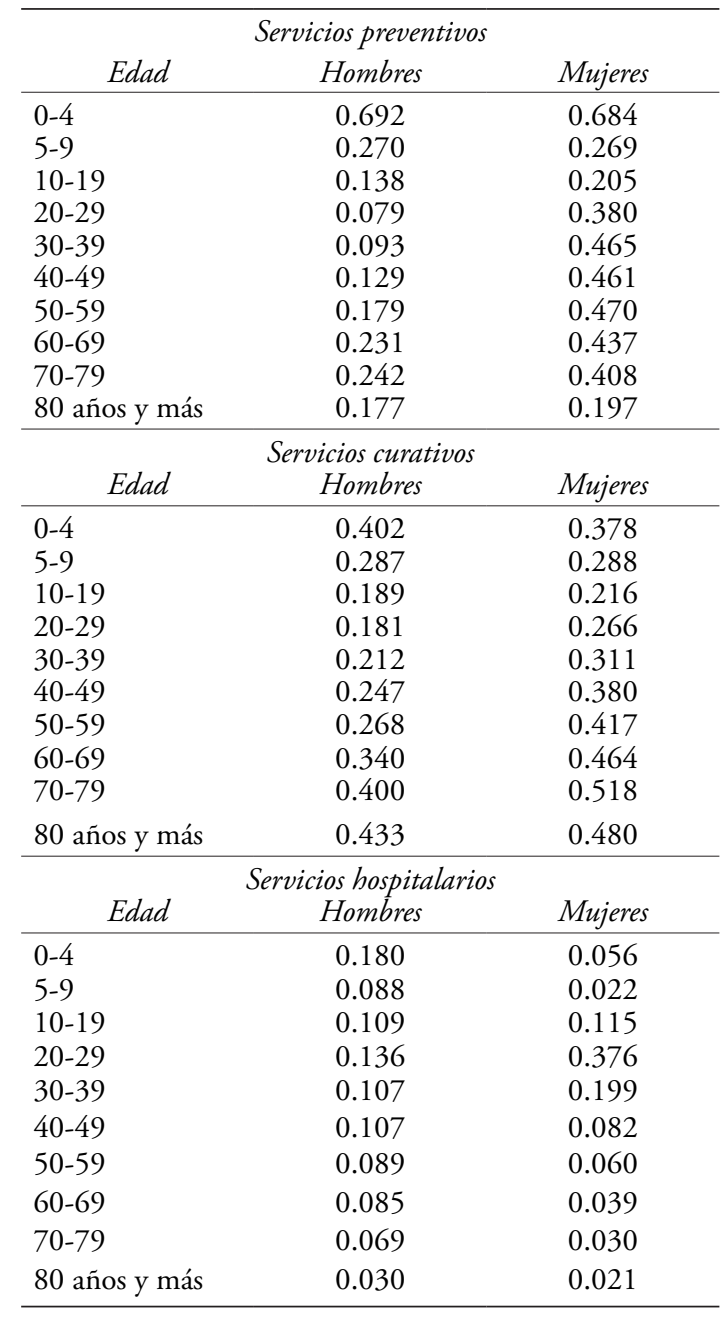

Fuente: Ensa-2000.

de asistencia. Cabe mencionar que esta tasa de asistencia varía de manera considerable si se clasifica por grupos de edades y género, así como por tipo de servicio médico (cuadro 4). Una vez que las transferencias se cargaron a cada individuo por uso de servicio médico de acuerdo con sus características demográficas, éstas se agregaron para obtener las trasferencias por familia, tanto totales como por tipo de servicio médico.

Finalmente, se comprobó que el monto estimado total de servicios públicos de salud otorgado a los hogares vía trasferencias, sí coincide con 
el gasto en salud pública reportado por las fuentes macroeconómicas. Antes de ello, es necesario mencionar la inexistencia en México de datos consolidados del gasto en salud, de modo que esta carencia de datos agregados oficiales dificulta la comparación. Además, algunas instituciones de salud destinan cuantiosos recursos a gastos que no son propios de este rubro, y es difícil distinguir entre gastos en salud y otros tipos de erogaciones.

A pesar de ello, se elaboró una serie de gastos en salud (cuadro 1), donde en el gasto del IMss se excluyeron los recursos destinados a pensiones. Al compararse esta serie con la estimación del monto de las transferencias en salud a través de las encuestas, se encontró una cantidad menor en el monto estimado vía encuestas. Entre las explicaciones de tal subestimación se encuentra la omisión de los altos gastos administrativos y de pensiones ${ }^{11}$ en la metodología empleada para estimar las transferencias, algo que parece totalmente lógico.

Por último, aunque la cantidad estimada de transferencias en salud mediante las encuestas podría ser la más adecuada, ya que es la que realmente reciben los hogares en servicios de salud y excluye los costos administrativos y burocráticos, también se debe reconocer que con frecuencia estos costos no pueden desaparecer tan fácilmente y muchas veces son intrínsecos al gasto en salud, y por tanto han de tomarse en cuenta a pesar de que sean ineficientes. En tal sentido, se procedió a ajustar los datos obtenidos en las encuestas por el factor de ajuste que surge de la comparación entre datos micro y macro (cuadro 5); así, una vez hecho dicho ajuste, los datos están preparados para su análisis de incidencia.

\section{Distribución de las transferencias públicas a los hogares}

Las transferencias per cápita registraron un aumento generalizado en todos los deciles entre 1984 y 1989, sin embargo, entre 1989 y 1996 hubo un importante descenso, explicado en buena medida por las restricciones presupuestales impuestas por la crisis de 1995, para después recuperarse en 2002. Por otro lado, la estructura de las transferencias per cápita es más o menos homogénea entre deciles, con un movimiento a favor de los primeros, ya que en el año 1984 todavía se mostraba una tendencia creciente en el monto de las trasferencias a medida que aumentaban los ingresos familiares. En perspectiva, mientras que en 1989 los individuos del primer decil recibían una cantidad considerablemente menor que el resto de familias, con el paso del tiempo se puede observar un mayor equilibrio en las transferencias para los años siguientes; de manera que el

${ }^{11}$ ISsSTe, Pemex, Sedena, etcétera. 


\section{Cuadro 5 \\ Gasto público en salud}

Análisis comparativo: ENIGH vs. cuentas públicas (millones de pesos corrientes)

\begin{tabular}{rrrc}
\hline Año & ENIGH & Cuentas nacionales & Cuentas nacionales-ENIGH \\
\hline 1984 & 414 & 817 & 1.97 \\
1989 & 9,010 & 14,081 & 1.64 \\
1996 & 44,900 & 58,710 & 1.31 \\
2002 & 93,700 & 180,847 & 1.93 \\
\hline
\end{tabular}

Nota: Para los años 1984 y 1989 se utilizó el gasto programable ejercido en el subsector salud federal, tomado de la cuenta de la hacienda pública Federal.

Fuente: Elaboración propia con datos de las ENIGH, Ensa y cuentas nacionales.

año 2002 presenta una mayor homogeneidad, aunque aún está lejos de ser totalmente equitativo (cuadro 6).

\section{Cuadro 6}

Transferencias monetarias ${ }^{\mathrm{a}}$ en salud, per cápita y por decil (pesos corrientes de 2002)

\begin{tabular}{ccccccccccc}
\hline Decil & 1 & 2 & 3 & 4 & 5 & 6 & 7 & 8 & 9 & 10 \\
\hline 1984 & 1,014 & 1,055 & 1,276 & 1,340 & 1,378 & 1,449 & 1,499 & 1,699 & 1,678 & 1,715 \\
1989 & 1,318 & 1,451 & 1,408 & 1,449 & 1,444 & 1,453 & 1,474 & 1,416 & 1,404 & 1,396 \\
1996 & 1,082 & 1,148 & 1,173 & 1,213 & 1,232 & 1,266 & 1,282 & 1,315 & 1,315 & 1,325 \\
2002 & 1,534 & 1,707 & 1,682 & 1,796 & 1,791 & 1,841 & 1,821 & 1,866 & 1,786 & 1,843 \\
\hline
\end{tabular}

${ }^{a}$ Con cargo a cada individuo, con base en el cálculo de precios sombra de las transferencias en especie por concepto de salud.

Fuente: Elaboración propia con datos de las ENIGH, Ensa y cuentas nacionales.

La tendencia hacia una mayor progresividad mostrada por las transferencias públicas en salud en los años de estudio, se hace evidente en la distribución porcentual por decil; donde se observa una ligera corrección en los 18 años que contempla el estudio, de manera que mientras en 1984 el último decil recibía alrededor de 2.6 veces más recursos que el primero, en el año 2002, aunque continuaba recibiendo mas transferencias, únicamente recibía 1.4 veces más (cuadro 7 ).

En suma, se observa un cambio en la estructura de las transferencias en salud durante los últimos 18 años. Este cambio ha propiciado una evolución desde una estructura donde $40 \%$ de las familias con mayores ingresos recibían una participación más que proporcional en el año 1984, hacia una estructura en la que de los deciles cuarto al octavo captan una proporción de transferencias en salud ligeramente mayor a la que tenían 


\section{Cuadro 7 \\ Distribución de las transferencias públicas en salud a las familiares mexicanas}

\begin{tabular}{clllrrrrrrc}
\hline Decil & 1 & 2 & 3 & \multicolumn{1}{c}{4} & \multicolumn{1}{c}{5} & \multicolumn{1}{c}{6} & 7 & 8 & 9 & 10 \\
\hline 1984 & 5.0 & 6.9 & 8.3 & 9.9 & 9.5 & 10.5 & 10.9 & 12.9 & 13.1 & 13.0 \\
1989 & 7.9 & 9.3 & 9.8 & 10.4 & 9.4 & 10.7 & 10.8 & 11.2 & 10.6 & 10.0 \\
1996 & 7.6 & 8.5 & 9.4 & 9.9 & 9.8 & 10.8 & 11.4 & 11.1 & 11.2 & 10.5 \\
2002 & 6.3 & 8.4 & 9.2 & 10.1 & 10.8 & 11.4 & 11.2 & 11.5 & 11.0 & 10.3 \\
\hline
\end{tabular}

Fuente: Elaboración propia con datos de las ENIGH, Ensa y cuentas nacionales.

en el primer año de estudio. Esto indica un ligero movimiento a favor hacia los deciles que representan las clases medias, en principio este ya es un cambio positivo, pero que se debe consolidar mediante un mayor beneficio hacia el 30\% de las familias con menores ingresos, quienes aún registran transferencias menos que proporcionales de las que les correspondería, simplemente por su peso demográfico (cuadro 8).

Dado que el sistema de procuración de salud no es universal, se analiza la incidencia por tipo de instituciones proveedoras de salud, así como por tipo de atención médica y zona geográfica. Llevando el análisis por tipo de atención, se encuentra que la salud preventiva presenta una estructura casi proporcional en las transferencias, con una ligera desventaja para los tres primeros deciles y pocos cambios en su estructura durante el tiempo. Por su parte, los servicios curativos también presentan casi una neutralidad, con una ligera desventaja para el 20\% de las familias con menores ingresos y con pocos cambios en el comportamiento de la estructura de transferencias en18 ańos.

En contraste, los servicios hospitalarios son los que han presentado un cambio mayor en las transferencias, ya que mientras en 1984 existía una clara tendencia a favorecer a las familias con mayores ingresos, en el año 2002 registran casi la misma proporción, si bien con una ligera ventaja para los deciles intermedios y todavía una menor participación de las familias con menos ingresos. Por lo anterior, a pesar de la mayor canalización de recursos hacia las familias más necesitadas que se ha dado durante el periodo de estudio, aún reciben menores trasferencias de las que les correspondería, de modo que es necesario reorientar y focalizar un poco más los recursos de salud hacia este tipo de familias, en especial al $20 \%$ con menores ingresos.

Finalmente, los servicios hospitalarios, que son los que tienen mayor valor agregado y por tanto mayores transferencias en términos monetarios, son también donde las familias con menores ingresos se encuentran en 


\section{Cuadro 8}

\section{Distribución porcentual de las transferencias públicas totales en salud por institución proveedora}

\begin{tabular}{|c|c|c|c|c|c|c|c|c|c|c|}
\hline Decil & 1 & 2 & 3 & 4 & 5 & 6 & 7 & 8 & 9 & 10 \\
\hline \multicolumn{11}{|c|}{ Total nacional } \\
\hline 1984 & 5.02 & 6.90 & 8.31 & 9.92 & 9.48 & 10.51 & 10.90 & 12.86 & 13.11 & 12.99 \\
\hline 1989 & 7.85 & 9.28 & 9.75 & 10.43 & 9.41 & 10.70 & 10.77 & 11.24 & 10.63 & 9.95 \\
\hline 1996 & 7.61 & 8.53 & 9.38 & 9.87 & 9.75 & 10.76 & 11.37 & 11.10 & 11.15 & 10.48 \\
\hline 2002 & 6.30 & 8.38 & 9.15 & 10.13 & 10.79 & 11.39 & 11.17 & 11.45 & 10.95 & 10.30 \\
\hline \multicolumn{11}{|l|}{ SSA } \\
\hline 1984 & 11.22 & 15.00 & 13.39 & 12.28 & 11.37 & 9.82 & 7.88 & 6.68 & 5.13 & 7.24 \\
\hline 1989 & 12.88 & 10.45 & 11.80 & 10.33 & 8.11 & 8.77 & 8.69 & 9.70 & 9.46 & 9.80 \\
\hline 1996 & 14.06 & 13.74 & 13.67 & 12.25 & 9.92 & 9.70 & 8.47 & 6.30 & 6.24 & 5.66 \\
\hline 2002 & 11.75 & 11.96 & 11.64 & 11.21 & 11.10 & 8.94 & 8.97 & 8.75 & 7.88 & 7.80 \\
\hline \multicolumn{11}{|l|}{ IMSS } \\
\hline 1984 & 0.72 & 1.20 & 6.15 & 8.45 & 9.55 & 11.76 & 12.60 & 18.89 & 16.54 & 14.15 \\
\hline 1989 & 4.34 & 9.30 & 8.14 & 10.62 & 10.89 & 12.34 & 12.12 & 12.56 & 10.47 & 9.22 \\
\hline 1996 & 0.53 & 3.41 & 5.54 & 8.40 & 10.80 & 13.11 & 14.54 & 16.30 & 14.08 & 13.29 \\
\hline 2002 & 0.82 & 5.53 & 7.29 & 10.42 & 11.72 & 14.63 & 13.77 & 13.17 & 12.14 & 10.52 \\
\hline \multicolumn{11}{|l|}{ ISSSTE } \\
\hline 1984 & 0.24 & 0.82 & 1.04 & 7.75 & 4.74 & 9.26 & 14.28 & 13.42 & 24.26 & 24.19 \\
\hline 1989 & 1.08 & 5.54 & 8.32 & 9.11 & 9.05 & 12.32 & 13.78 & 12.93 & 15.12 & 12.75 \\
\hline 1996 & 0.08 & 0.54 & 1.66 & 3.49 & 5.63 & 7.74 & 14.42 & 16.87 & 25.61 & 23.97 \\
\hline 2002 & 0.04 & 0.85 & 3.88 & 3.83 & 5.98 & 10.13 & 12.59 & 18.70 & 21.28 & 22.72 \\
\hline \multicolumn{11}{|c|}{ Pemex-Sedena-Secmar } \\
\hline 1996 & 0.82 & 0.21 & 0.00 & 1.67 & 1.71 & 9.48 & 16.26 & 16.67 & 22.82 & 30.34 \\
\hline 2002 & 0.00 & 2.97 & 7.93 & 3.29 & 3.22 & 17.82 & 9.42 & 17.42 & 24.71 & 13.77 \\
\hline
\end{tabular}

Fuente: Elaboración propia con base en las ENIGH, Ensa-2000 y diversos informes presidenciales.

mayor desventaja. Para obtener un análisis más minucioso, se hizo un breve estudio por tipo de institución proveedora (cuadros 8-11).

\subsection{Secretaría de Salud}

El conjunto de servicios de salud prestados por la Secretaría de Salud (ss) son totalmente favorables a las familias con bajos ingresos para todos los años estudiados, si bien resalta el año 2002, donde se observa un aumento de las transferencias para los de mayores ingresos. Una hipótesis para explicar este fenómeno es el deterioro general del nivel de vida, tras la crisis de 1995, que implicó la pérdida de empleos en el sector formal de 


\section{Cuadro 9}

Distribución de las transferencias públicas en servicios preventivos por institución proveedora

\begin{tabular}{lcrrrrrrrrr}
\hline \multicolumn{1}{c}{ Decil } & 1 & 2 & \multicolumn{1}{c}{3} & \multicolumn{1}{c}{4} & 5 & 6 & 7 & 8 & 9 & 10 \\
\hline \multicolumn{2}{l}{ Total nacional } & & & & & & & & & \\
1984 & 7.61 & 9.75 & 9.94 & 11.36 & 10.03 & 10.13 & 10.03 & 10.51 & 10.32 & 10.32 \\
1989 & 9.07 & 9.75 & 10.43 & 10.70 & 9.48 & 10.50 & 10.09 & 10.63 & 10.09 & 9.27 \\
1996 & 9.26 & 9.73 & 10.17 & 10.22 & 9.76 & 10.28 & 10.60 & 10.10 & 10.28 & 9.59 \\
2002 & 7.67 & 9.10 & 9.71 & 10.26 & 10.65 & 10.92 & 10.76 & 10.81 & 10.59 & 9.54 \\
SSA & & & & & & & & & & \\
1984 & 12.42 & 15.70 & 13.40 & 10.55 & 11.29 & 9.23 & 7.80 & 5.92 & 4.83 & 6.50 \\
1989 & 13.94 & 10.59 & 12.37 & 10.55 & 8.31 & 8.66 & 8.29 & 9.38 & 8.86 & 9.04 \\
1996 & 15.54 & 14.48 & 13.97 & 11.99 & 9.87 & 9.10 & 7.92 & 6.06 & 5.88 & 5.20 \\
2002 & 13.23 & 12.44 & 11.65 & 11.35 & 10.56 & 8.78 & 8.80 & 8.33 & 7.49 & 7.36 \\
IMSS & & & & & & & & & & \\
1984 & 0.91 & 1.44 & 6.49 & 9.29 & 9.75 & 12.28 & 12.95 & 18.74 & 15.54 & 12.61 \\
1989 & 5.00 & 10.02 & 8.58 & 10.77 & 11.09 & 12.31 & 11.39 & 11.80 & 10.30 & 8.75 \\
1996 & 0.65 & 3.93 & 5.97 & 9.14 & 10.92 & 13.13 & 14.15 & 15.38 & 13.61 & 13.13 \\
2002 & 0.89 & 5.86 & 7.92 & 10.41 & 12.17 & 14.41 & 13.49 & 12.79 & 12.29 & 9.78 \\
ISSSTE & & & & & & & & & & \\
1984 & 0.40 & 0.95 & 1.24 & 8.95 & 4.91 & 9.69 & 13.68 & 13.46 & 23.95 & 22.77 \\
1989 & 1.38 & 6.17 & 8.48 & 10.24 & 9.11 & 12.31 & 13.32 & 12.56 & 14.13 & 12.31 \\
1996 & 0.09 & 0.60 & 1.64 & 3.68 & 5.92 & 7.71 & 14.83 & 16.85 & 25.39 & 23.29 \\
2002 & 0.06 & 1.41 & 4.37 & 3.85 & 6.06 & 10.10 & 12.53 & 18.25 & 21.60 & 21.78 \\
Pemex-Sedena-Secmar & & & & & & & & \\
1996 & 1.04 & 0.27 & 0.00 & 1.60 & 1.73 & 10.79 & 15.38 & 16.84 & 23.45 & 28.90 \\
2002 & 0.00 & 2.80 & 7.78 & 3.01 & 2.99 & 18.15 & 8.83 & 16.80 & 24.70 & 14.94 \\
\hline
\end{tabular}

Fuente: Elaboración propia con base en las ENIG, Ensa-2000 y diversos informes presidenciales.

la economía y, por tanto, del derecho a servicios de salud por parte de las instituciones de seguridad social tradicionales, de forma que las clases medidas aumentaron la demanda de servicios prestados por la ss, los cuales en un principio se diseñaron para la población que no gozaba de ningún tipo de cobertura médica y con un bajo nivel de ingresos.

A pesar de ello, el buen nivel de progresividad de los servicios de salud de la ss es la constante para todo los tipos de cuidados médicos: preventivos, curativos y hospitalarios, si bien estos últimos registran una menor progresividad, casi proporcional. En conjunto, y de manera simultánea, la estructura de las participaciones ha tenido pocos cambios durante los años que comprende el estudio. 


\section{Cuadro 10}

\section{Distribución de las transferencias públicas en servicios curativos por institución proveedora}

\begin{tabular}{lccccccccccc}
\hline Decil & 1 & 2 & 3 & \multicolumn{1}{c}{4} & 5 & 6 & 7 & 8 & 9 & 10 \\
\hline \multicolumn{2}{l}{ Total nacional } & & & & & & & & & \\
1984 & 7.20 & 8.91 & 9.13 & 10.68 & 9.66 & 10.26 & 10.40 & 11.33 & 11.35 & 11.09 \\
1989 & 8.48 & 9.31 & 9.85 & 10.75 & 9.49 & 10.84 & 10.39 & 10.93 & 10.39 & 9.58 \\
1996 & 8.63 & 8.97 & 9.48 & 9.82 & 9.75 & 10.53 & 10.95 & 10.72 & 10.92 & 10.23 \\
2002 & 7.29 & 8.65 & 9.44 & 9.94 & 10.51 & 11.22 & 10.94 & 11.01 & 10.94 & 10.08 \\
SSA & & & & & & & & & & \\
1984 & 12.75 & 15.45 & 13.02 & 12.35 & 11.16 & 9.54 & 7.99 & 6.01 & 5.05 & 6.70 \\
1989 & 13.76 & 10.37 & 11.99 & 10.55 & 8.36 & 8.78 & 8.31 & 9.68 & 9.01 & 9.19 \\
1996 & 15.71 & 14.25 & 13.69 & 11.00 & 9.86 & 9.30 & 8.01 & 6.16 & 5.90 & 5.25 \\
2002 & 13.53 & 12.46 & 11.74 & 11.16 & 10.43 & 8.85 & 8.79 & 8.05 & 7.55 & 7.43 \\
IMSS & & & & & & & & & & \\
1984 & 0.98 & 1.61 & 6.12 & 8.91 & 9.38 & 12.01 & 12.83 & 19.23 & 15.82 & 13.11 \\
1989 & 5.04 & 9.48 & 8.20 & 10.86 & 10.77 & 12.58 & 11.61 & 11.81 & 10.52 & 9.13 \\
1996 & 0.64 & 3.68 & 5.67 & 8.71 & 10.91 & 13.23 & 14.20 & 15.62 & 13.94 & 13.42 \\
2002 & 0.92 & 5.57 & 7.77 & 10.11 & 11.99 & 14.48 & 13.47 & 12.98 & 12.52 & 10.20 \\
ISSSTE & & & & & & & & & & \\
1984 & 0.55 & 1.06 & 1.36 & 8.44 & 4.74 & 8.99 & 13.89 & 13.26 & 24.75 & 22.97 \\
1989 & 1.35 & 6.25 & 8.46 & 10.14 & 8.97 & 12.25 & 13.35 & 12.54 & 14.29 & 12.40 \\
1996 & 0.08 & 0.67 & 1.63 & 3.62 & 5.93 & 7.81 & 14.47 & 17.05 & 25.41 & 23.35 \\
2002 & 0.05 & 1.45 & 4.05 & 4.05 & 5.88 & 10.20 & 12.40 & 18.14 & 21.40 & 22.39 \\
Pemex-Sedena-Secmar & & & & & & & & \\
1996 & 1.23 & 0.34 & 0.00 & 1.44 & 1.75 & 9.56 & 15.94 & 16.95 & 23.90 & 28.82 \\
2002 & 0.00 & 3.20 & 6.22 & 2.75 & 3.07 & 17.97 & 9.83 & 18.12 & 23.42 & 15.43 \\
\hline
\end{tabular}

Fuente: Elaboración propia con base en las ENIGH, Ensa-2000 y diversos informes presidenciales.

\subsection{IMSS}

Las transferencias agregadas en salud a través del IMss han evolucionado desde un alto nivel de concentración a favor de las familias con mayores ingresos, hacia un nivel más homogéneo, aunque todavía favorable a los ingresos medios y altos. Tomando 2002 como referencia, 30\% de las familias con menores ingresos registraron una participación menos que proporcional, mientras que los deciles intermedios estuvieron ligeramente por arriba de la proporcionalidad. Sin embargo, la evolución durante el periodo de estudio ha sido continua, aunque en 1989 se presentó la estructura menos desigual de los cuatro años contemplados. Para ello hay 


\section{Cuadro 11}

Distribución de las transferencias públicas en servicios hospitalarios por institución proveedora

\begin{tabular}{lcccrrrrrrr}
\hline \multicolumn{1}{l}{ Decil } & 1 & 2 & 3 & \multicolumn{1}{c}{4} & \multicolumn{1}{c}{5} & \multicolumn{1}{c}{6} & 7 & 8 & 9 & 10 \\
\hline \multicolumn{2}{l}{ Total nacional } & & & & & & & & & \\
1984 & 4.32 & 6.18 & 7.94 & 9.60 & 9.38 & 10.60 & 11.14 & 13.43 & 13.77 & 13.65 \\
1989 & 7.60 & 9.17 & 9.67 & 10.32 & 9.42 & 10.73 & 10.89 & 11.39 & 10.73 & 10.08 \\
1996 & 7.31 & 8.34 & 9.27 & 9.82 & 9.76 & 10.85 & 11.51 & 11.24 & 11.28 & 10.62 \\
2002 & 6.05 & 8.24 & 9.10 & 10.17 & 10.83 & 11.43 & 11.23 & 11.56 & 10.96 & 10.43 \\
sSA & & & & & & & & & & \\
1984 & 10.63 & 14.72 & 13.45 & 12.10 & 11.43 & 10.04 & 7.89 & 7.00 & 5.23 & 7.51 \\
1989 & 12.66 & 10.43 & 11.71 & 10.29 & 8.07 & 8.79 & 8.78 & 9.73 & 9.58 & 9.96 \\
1996 & 13.71 & 13.59 & 13.63 & 12.20 & 9.94 & 9.03 & 9.60 & 6.34 & 6.34 & 5.74 \\
2002 & 11.43 & 11.91 & 11.57 & 11.20 & 11.19 & 8.97 & 9.01 & 8.87 & 7.96 & 7.89 \\
IMSs & & & & & & & & & & \\
1984 & 0.68 & 1.14 & 6.12 & 8.30 & 9.55 & 11.66 & 12.55 & 10.09 & 16.70 & 14.41 \\
1989 & 4.19 & 9.22 & 8.08 & 10.59 & 10.87 & 12.33 & 12.25 & 12.71 & 10.47 & 9.28 \\
1996 & 0.51 & 3.33 & 5.47 & 8.27 & 10.79 & 13.10 & 14.63 & 16.44 & 14.14 & 13.32 \\
2002 & 0.80 & 5.50 & 7.17 & 10.44 & 11.65 & 14.70 & 13.81 & 13.23 & 12.07 & 10.63 \\
IsssTE & & & & & & & & & & \\
1984 & 0.19 & 0.78 & 0.99 & 7.56 & 4.72 & 9.25 & 14.89 & 13.44 & 24.25 & 24.43 \\
1989 & 1.01 & 5.39 & 8.29 & 8.88 & 9.03 & 12.36 & 13.91 & 13.02 & 15.32 & 12.80 \\
1996 & 0.08 & 0.51 & 1.67 & 3.45 & 5.55 & 7.73 & 14.34 & 16.84 & 25.69 & 24.15 \\
2002 & 0.04 & 0.72 & 3.80 & 3.80 & 5.97 & 10.13 & 12.62 & 18.79 & 21.21 & 22.90 \\
Pemex-Sedena-Secmar & & & & & & & & \\
1996 & 0.77 & 0.20 & 0.00 & 1.69 & 1.72 & 9.36 & 16.38 & 16.54 & 22.65 & 30.68 \\
2002 & 0.00 & 2.96 & 7.49 & 3.36 & 3.26 & 17.76 & 9.45 & 17.41 & 21.77 & 13.55 \\
\hline
\end{tabular}

Fuente: Elaboración propia con base en las ENIGH, Ensa-2000 y diversos informes presidenciales.

una explicación que puede ser muy plausible, y es que se ha contabilizado el programa IMss-Coplamar para ese año, el cual está enfocado sobre todo a la población de escasos recursos y residente mayoritariamente en áreas rurales y pequeñas áreas urbanas.

En cuanto al tipo de atención médica, la preventiva y la curativa muestran un cambio a favor de los ingresos intermedios, en claro detrimento tanto del primer como del último decil, con el agravante de que $10 \%$ de las familias más pobres no llega a recibir en ninguno de los dos casos el $1 \%$ de las trasferencias. En cuanto a los servicios hospitalarios, ha habido una evolución favorable hacia el primer $50 \%$ de la población, aunque con un beneficio sólo marginal al primer decil. En suma, aunque hoy las transferencias son más homogéneas que nunca, son las clases medias las más beneficiadas, puesto que han ganado mayor presencia. 


\subsection{ISSSTE}

Aquí es donde existe la mayor concentración de las transferencias en salud a favor de los hogares con mayores ingresos, beneficiando sobre todo al $40 \%$ más rico. Sin embargo, debe decirse que aunque durante el periodo estudiado ha habido algunos cambios hacia una menor concentración, éstos no han sido sustanciales. La razón principal se encuentra en que dichos servicios de salud atienden a una población muy concreta ${ }^{12}$ y que difícilmente cambia su perfil con el tiempo. Cuando se evalúan estos servicios por tipo de atención médica, se observa que en las tres categorías se reproduce la misma concentración que en el agregado, lo que implica pocos cambios de la estructura en el tiempo, y un patrón muy claro que favorece a $50 \%$ de la población con más ingresos.

\subsection{Petróleos Mexicanos (Pemex), Secretaría de la Defensa Nacional (Sedena), Secretaría de Marina (Secmar)}

De manera general, los servicios de salud prestados por estas instituciones registran una importante desconcentración en los dos últimos años contemplados en el análisis. A pesar de ello, estos servicios siguen beneficiando con mucha diferencia a la mitad más rica de la población. Cuando se analiza el tipo de atención médica por decil, se reproduce prácticamente el mismo patrón, con una pérdida importante para el $10 \%$ con mayores ingresos y mayor beneficio para el resto, especialmente los deciles medios-altos (sexto a noveno), puesto que $10 \%$ de las familias más pobres están literalmente ausentes en los servicios que prestan estas instituciones. Otra característica es que el perfil de los beneficiados es en su mayoría urbano, mientras que en el ámbito rural, prácticamente $50 \%$ de las familias con menores ingresos no recibe transferencia alguna por parte de estas instituciones.

Resumiendo la estructura de los beneficios por parte de las instituciones proveedoras de salud, se puede decir que la ss, y en menor medida el IMSs, tienden a beneficiar a una población con menores recursos. Los cuidados preventivos son más importantes en la ss y los hospitalarios en el IMss; sobre todo hacia la población urbana en el caso del IMss y la población rural en el de la ss. Por su parte, instituciones como el Issste, Pemex, la Sedena y la Secmar, atienden a un nicho específico de la población que les confiere una procuración de salud más orientada a familias de ingresos medios-altos y altos, en especial urbano y con mayor énfasis en servicios curativos y hospitalarios (cuadros 9-11).

${ }^{12}$ Trabajadores al servicio del Estado. 


\section{Progresividad de las transferencias}

Existe una abundante literatura con igual gran cantidad de índices de desigualdad, algunos simplemente contienen datos estadísticos y otros también incluyen juicios de valor, de esta manera surge una importante disyuntiva cuando se trata de elegir un índice, la cual consiste en buscar un equilibrio entre atractivo estadístico y facilidad de interpretación con contenido normativo.

Las propiedades de los índices son un buen indicador a la hora de elegir alguno, por ejemplo Shorroks (1983) apunta que el grado de sensibilidad a las transferencias es la característica más distintiva que debe tener un índice.

Los índices elegidos para el presente análisis han de cumplir con el principio de transferencia débil, el cual se da cuando una transferencia a un individuo con ingreso $(y)$, por parte de otro individuo más rico con ingreso $y+\delta$, donde dicha transferencia es inferior a $\delta / 2$, hace que la desigualdad disminuya.

El teorema sostiene: "Supóngase que la distribución del ingreso en el estado social A pueda ser alcanzado por una simple redistribución del ingreso en el estado social $\mathrm{B}$ (manteniéndole siempre constante el ingreso) y la curva de Lorenz para A se ubica totalmente dentro de la de B. Entonces, en la medida que se satisfaga el principio de transferencia débil, la medida de desigualdad siempre indicará un estrictamente menor nivel de desigualdad para el estado A que para el estado B" (Lambert, 2001: 55).

Otro factor que se tomó en cuenta en la elección de los índices de concentración y de Kakwani para medir la progresividad de las transferencias en salud, es su fácil interpretación, ya que tienen como base el índice de Gini y la curva de Lorenz.

Respecto a la interpretación, para los casos donde hay transferencias, los menores índices de concentración implican mejor distribución para los ingresos más bajos, lo que implica la misma lógica del coeficiente de Gini; de hecho, cuando se tornan negativos, indican que los recursos transferidos se concentran en las familias con menores ingresos y se dice que la transferencia en términos absolutos es totalmente redistributiva.

Una vez explicadas brevemente la lógica y justificación de los índices a utilizar, se procede a su interpretación. Así, el coeficiente de concentración de las trasferencias totales en salud para los diferentes años de estudio, muestra una tendencia decreciente, lo que implica que este tipo de trasferencias sean en el último año de análisis casi neutrales, después de mostrar en un inicio cierta preferencia hacia los mayores ingresos. Sin embargo, por tipo de institución proveedora de salud, existen divergencias importantes (cuadro 12). 
Los servicios de salud adscritos a la Secretaría de Salud son los únicos que registran índices de concentración negativos para las cuatro encuestas analizadas, el comportamiento de las transferencias va desde una ligera progresividad cercana a la neutralidad en 1984 , hasta una clara progresividad para el año 2002, con un coeficiente de concentración de -0.1136.

El coeficiente de concentración de IMss ha tenido un comportamiento errático con respecto al año de inicio del análisis, con una disminución en 1989 explicado ya por la posible inclusión del Programa Imss-Coplamar, un aumento en 1996 y finalmente una ligera caída en el año 2002, de manera que ha permanecido casi invariable en 18 años, si acaso aumentó ligeramente la ventaja hacia los altos ingresos. Por su parte, el ISSSTE muestra los más altos coeficientes de concentración, con un incremento de $27 \%$ durante los ańos estudiados, lo que significa que los servicios de esta institución han tendido a concentrarse en las familias con mayores ingresos, empeorando el efecto redistributivo que pudiesen tener sus servicios. A manera de conclusión, se observan dos claros patrones en cuanto a progresividad para los servicios de salud: por un lado los servicios prestados por la Secretaría de Salud, que son los que benefician a la población abierta y muestran una tendencia hacia la progresividad; y por otro, los servicios prestados a la población asegurada por las dos principales instituciones públicas de seguridad social, los cuales muestran una claro patrón regresivo. Aunque cuando se considera el sistema completo, se advierte un ligero sesgo hacia la progresividad, conducido y soportado en su totalidad por los servicios universales prestados por la ss, los cuales benefician a la población abierta.

Con base en el índice de Kakwani, el cual trata de cuantificar la desproporcionalidad de las transferencias en salud y, por tanto, la distancia entre la curva de Lorenz del ingreso disponible y de concentración, se pretende capturar su nivel de progresividad.

Las transferencias en salud también se muestran bastante progresivas, incluso mayores que en educación, con altos valores en el índice de Kakwani y un aumento de $13.6 \%$ durante el periodo analizado. Sin embargo, cuando se observa la evolución por institución proveedora aparecen diferencias importantes. Mientras los servicios de la ss registran un índice de 0.621 y un aumento de $13 \%$ en su valor, los ofrecidos por el ISSSTE muestran un índice de apenas 0.036 y una caída en su valor de $72 \%$; en tanto los ofrecidos por el IMSs se encuentran en valores intermedios. De esta manera existe un claro contraste entre la progresividad y evolución por tipo de institución, donde la alta progresividad de los servicios de la ss se ven contrarrestados por la nula progresividad del ISSSTE (cuadro 12). 


\section{Cuadro 12 \\ Transferencias en salud por tipo de institución Índices de concentración}

\begin{tabular}{lcccc}
\hline \multicolumn{1}{c}{ Tipo de transferencia } & 1984 & 1989 & 1996 & 2002 \\
\hline Total & 0.1096 & 0.0424 & 0.0615 & 0.0648 \\
& -0.0060 & 0.0035 & 0.0028 & 0.0027 \\
SSA & -0.0697 & -0.0574 & -0.1237 & -0.1136 \\
& 0.0131 & 0.0072 & 0.0052 & 0.0050 \\
IMSS & 0.1752 & 0.0868 & 0.2264 & 0.1983 \\
& 0.0013 & 0.0087 & 0.0078 & 0.0067 \\
ISSSTE & 0.3690 & 0.2277 & 0.4786 & 0.4720 \\
& 0.0207 & 0.0141 & 0.0116 & 0.0108 \\
\hline \multirow{2}{*}{ Tipo de Transferencia } & 1984 dices de & Kakwani & & \\
\hline Total & 0.3906 & 0.5441 & 0.4616 & 0.4436 \\
& 0.0104 & 0.0140 & 0.0107 & 0.0054 \\
SSA & 0.5612 & 0.6438 & 0.6467 & 0.6219 \\
& 0.0130 & 0.0153 & 0.0114 & 0.0068 \\
IMss & 0.3250 & 0.4996 & 0.2967 & 0.3101 \\
& 0.0158 & 0.0162 & 0.0129 & 0.0082 \\
IsssTE & 0.1311 & 0.3566 & 0.0444 & 0.0364 \\
& 0.0228 & 0.0195 & 0.0156 & 0.0118 \\
\hline
\end{tabular}

Fuente: Elaboración propia con información de las ENIGH.

\section{Conclusiones}

En años recientes, la participación del gasto público en salud como proporción del рів destinado a la población abierta (cobertura universal) ha aumentado, de manera que se ha triplicado en relación con 18 años atrás (cuadro 2), y se ha intentado focalizar el gasto hacia sectores más necesitados mediante la descentralización en la administración y gasto de los recursos en el ámbito regional, en donde se han utilizado criterios de asignación de recursos con base en los indicadores de salud locales.

Por tanto, el comportamiento de las transferencias en salud ha evolucionado de manera positiva en los últimos años, destacando sobre todo la cobertura proporcionada por los servicios del la ss. De hecho, estos servicios son los responsables en gran medida de los buenos resultados del sector salud, ya que instituciones como el ISSSTE muestran un comportamiento más bien nulo en su cobertura de los estratos económicos más bajos. Por su parte, el IMss también ha mejorado la progresividad de sus servicios, aunque en menor medida que los de la Secretaría de Salud.

De acuerdo con el análisis del nivel de progresividad para los diferentes subsistemas públicos de salud, es evidente que la creación de un siste- 
ma público único proveedor de salud sería la solución para dirimir las diferencias existentes, ello conllevaría a un sistema más equitativo donde los estratos de ingreso más desfavorecidos se beneficiarían de manera más proporcional, ya que mejoraría la cobertura y desaparecerían las todavía amplias discrepancias en los montos de transferencias existentes entre los diferentes subsistemas, con las repercusiones que pudiera traer en la calidad del servicio.

En el caso de un escenario falto de reformas, al menos se exige una creciente financiación del sistema ss con transferencias públicas, para expandir su cobertura y elevar la calidad de sus servicios, ya que se ha visto que la población más beneficiada por los servicios de salud de la ss se compone de los sectores más empobrecidos de la sociedad.

Recapitulando: el sistema de salud necesita una reforma integral, para universalizar los servicios y unificar las diferentes instituciones públicas proveedoras de salud. De esta manera se eliminará la duplicidad de servicios, se aprovecharán las economías de escala y de ámbito y se ahorraran recursos. Así, se eliminarán de manera más contundente las diferencias en la equidad de acceso a los recursos sanitarios, entre áreas rurales y urbanas, entre clases sociales y entre regiones, se ahorrarán recursos y la duplicidad de esfuerzos y, lo que es más importante, se elevaría el nivel de bienestar general de la población.

\section{Anexo metodológico}

\section{A.1. Curva de Lorenz}

Es uno de los instrumentos de análisis de la desigualdad de la renta más básicos que existen, pero también es uno de los más ilustrativos y prácticos, especialmente cuando se trata de comparar diferentes distribuciones de la renta $f(y)$, ya que estandariza el nivel de renta para su medición.

La idea básica es ordenar las unidades de ingreso por su nivel de magnitud, empezando por el nivel más bajo y graficando contra la proporción acumulativa de la población que le corresponde, en ambos casos, tanto el ingreso como la población han de normalizarse, de forma que tomen valores que van de 0 a 1 . Así, si existiese un distribución del ingreso muy desigual, los cuantiles (porciones) poblacionales con mayor ingreso recibirán una renta más que proporcional al tamaño de su población, mientras que los cuantiles menores recibirán una renta menos que proporcional. De esta manera, ante el hipotético caso de una total igualdad en la distribución del ingreso, cada cuantil poblacional recibirá exactamente el mismo cuantil de renta que le corresponde, donde cada individuo tendría el ingreso promedio $(\mu)$ y la curva de Lorenz sería una línea de $45^{\circ}$. 


\section{Figura A.I}

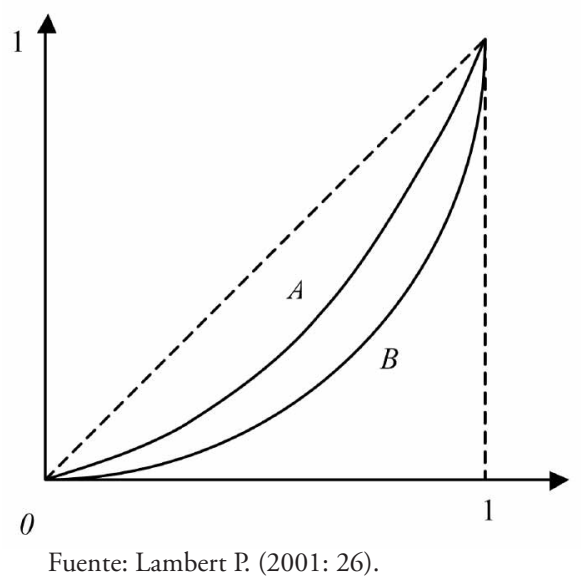

Es fácil comparar en la figura A.I dos distribuciones de ingreso familiar, la curva $\mathrm{A}$ es menos desigual que la $\mathrm{B}$, ya que está más cerca de la línea de igualdad.

En el caso de que la curva de Lorenz para una función continua sea $L(p), 0 \leq p \leq 1$, el ingreso del primer 100p por ciento de unidades de ingreso es $N z \int_{0}^{z} y f(y) d y=N \mu$, de manera que la curva de Lorenz $L(p)$ en el p-valor se define como:

$$
p=F(z) \Rightarrow L(p)=\int_{0}^{z} \frac{y f(y) d y}{\mu}
$$

\section{A.2. Curva de concentración}

Cuando se grafican porciones de una variable $x$ contra quintiles en la distribución de otra $y$, se llama curva de concentración y se compara con la curva de Lorenz, por lo que puede situarse por arriba o debajo de ésta, de modo que la diferencia entre ambas captura las diferencias en la clasificación de las distribuciones. Éstas son muy usadas en el análisis de los efectos distributivos de los impuestos y transferencias públicas. Debe decirse que en las curvas de concentración no se ordenan los valores de la variable en cuestión de menor a mayor, sino simplemente se mantiene el orden preestablecido de la variable de referencia, la cual en la mayoría de las veces es el ingreso. 


\section{A.3. Coeficiente de concentración}

Se le llama al área que se encuentra entre la curva de concentración y la línea de $45^{\circ}$, de manera análoga a como lo hace el coeficiente de Gini. Sus valores se encuentran entre - 1 para concentraciones sobre los ingresos más bajos de la variable en cuestión, $\mathrm{y}+1$ cuando la concentración se acentúa en los estratos con más ingresos, mientras que 0 indica una distribución neutral, lo que implica que cada persona o decil presenta una misma participación en la variable. Así, para el caso donde se analizan los efectos que tiene una transferencia determinada sobre el ingreso, el coeficiente de concentración para el ingreso postransferencia es:

$$
C_{Y+B}=1-2 \int_{0}^{1} L_{Y+B}(p) d p
$$

Y para las transferencias es:

$$
C_{B}=1-2 \int_{0}^{1} L_{B}(p) d p
$$

Analizando el efecto que una transferencia tiene sobre el ingreso, se define a la curva de Lorenz pretransferencia $L_{B}(p)$ como:

$$
p=F(z) \Rightarrow L(p)=\int_{0}^{z} \frac{y f(y) d y}{\mu}
$$

Mientras que la curva de concentración postransferencia $L_{Y+B}$ se define:

$$
p=F(z) \Rightarrow L_{Y+B}(p)=\int_{0}^{z} \frac{[y-b(y)] f(y) d y}{\mu(1-g)}
$$

La curva de concentración de transferencia $L_{B}$ se expresa:

$$
p=F(z) \Rightarrow L_{B}(p)=\int_{0}^{z} \frac{[b(y) f(y) d y}{\mu g}
$$

Donde la relación de las tres curvas es:

$$
L_{y}=g L_{B}+(1-g) L_{Y+B}
$$

Lo cual muestra que la curva de Lorenz $L_{Y}$ del ingreso pretransferencia es un promedio ponderado de las curvas de concentración de transferencias $L_{B}$ y del ingreso postransferencia $L_{Y-B}$. Por tanto, 


$$
L_{Y+B} \geq L_{y} \Leftrightarrow L_{B} \leq L_{y}
$$

Esta desigualdad indica que para que el ingreso postransferencia sea más equitativo que el ingreso pretransferencia, la transferencia sobre el ingreso deberá ser más equitativo que el ingreso pretransferencia

\section{A.4. Indice de Kakwani y progresividad}

La pregunta que surge ahora es cómo capturar esa nueva desproporcionalidad creada por una transferencia progresiva y su efecto redistributivo en el ingreso. En este sentido, un índice de progresividad tiene la ventaja de capturar en un número la estructura de transferencias $b(y)$ y la distribución de la renta pre $f(y)$ y post $f^{\prime}(y)$ transferencias.

La desproporcionalidad de un impuesto o transferencia progresiva se aprecia en la distancia existente entre las curvas $L_{Y}$ y $L_{B}$, de manera que a mayor separación entre ellas mayor será el efecto de desproporcionalidad. Esta idea se complementa con lo señalado por Musgrave y Tun (1948), para quienes entre más desigual sea la distribución del ingreso antes de una transferencia progresiva, más fuerte será su efecto ecualizador.

De esta manera surge el índice de Kakwani, el cual mide la progresividad en términos de la separación de las curvas $L_{Y}$ y $L_{B}$, a través de un índice que calcula dos veces el área existente entre ambas curvas.

$$
\Pi^{K}=2 \int_{0}^{1}\left[L_{Y}(p)-L_{B}(p)\right] d p
$$

También expresado como

$$
\prod^{K}=G_{B}-C_{Y}
$$

Así, $\prod^{K}$ aumentará si la progresividad de una transferencia se incrementa para cada nivel de ingreso, mientras la distribución del ingreso pretransferencia permanezca constante, aumentando así la progresividad efectiva.

Debe decirse que los límites de este índice dependerán de la desigualdad de la distribución pretransferencia, y es $\left(-1+G_{y}\right)$ el límite inferior y $\left(1+G_{y}\right)$ el límite superior. 


\section{Anexo gráfico}

\section{Gráfica I}

\section{Distribución de las transferencias públicas en salud} a las familias, 2002

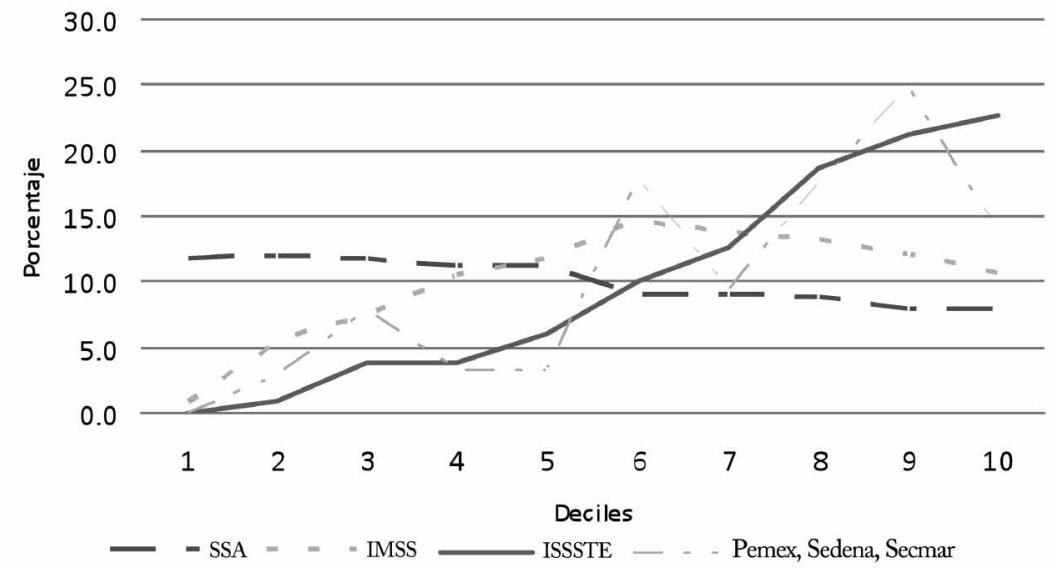

Fuente: Elaboración propia con datos de la ENIGH-2002 y ENSA.

\section{Bibliografía}

IMSS (Instituto Mexicano del Seguro Social) (2005), Población derechohabiente, Cuaderno de memoria estadística, IMss, México.

INEGI (Instituto Nacional de Estadística, Geografía e Informática) (1986), Aspectos metodológicos de la Encuesta de Ingreso y Gasto de los Hogares de 1984, INEGI, México.

INEGI (Instituto Nacional de Estadística, Geografía e Informática) (1991), Aspectos metodológicos de la Encuesta de Ingreso y Gasto de los Hogares de 1989, INEGI, Aguascalientes.

INEGI (Instituto Nacional de Estadística, Geografía e Informática) (1998), Aspectos metodológicos de la Encuesta de Ingreso y Gasto de los Hogares de 1996, INEGI, Aguascalientes.

INEGI (Instituto Nacional de Estadística, Geografía e Informática) (2004), Aspectos metodológicos de la Encuesta Ingreso Gasto de los Hogares de 1984, INEgi, Aguascalientes. 
INEGI (Instituto Nacional de Estadística, Geografía e Informática) (2004), Sistema de Cuentas Nacionales, INEGI, Aguascalientes.

INSP (Instituto Nacional de Salud Pública) (2000), “Encuesta Nacional de Salud 2000", InsP, Cuernavaca.

Lambert, P. (2001), The Distribution and Redistribution of Income, Manchester University Press, Manchester.

Musgrave, Richard y Thin Tun (1948), "Progressive taxation in an inflationary economy", Journal of Political Economy, 56, University of Chicago, Chicago, pp. 498-514.

oCDE (Organización para la Cooperación y el Desarrollo Económico (1997), Economic Survey of Mexico 1997, ocde, París.

Primer Informe Presidencial, Ernesto Zedillo (1995), "Anexo Estadístico", Presidencia de la República, México.

Sexto Informe Presidencial, Ernesto Zedillo (2000), "Anexo Estadístico", Presidencia de la República, México.

Scott-Andretta, John (2000), Progresa: una evaluación preliminar, Centro de Investigación y Docencia Económicas, México.

Scott-Andretta, John (2004), "Transferencias públicas ( y otros ingresos) en especie en la medición de la pobreza”, Documento de Trabajo núm. 301, Centro de Investigación y Docencia Económicas, México.

Scott-Andretta, John (2004), "Desigualdad en salud y en los recursos para la salud en México", Documento de Trabajo núm. 302, Centro de Investigación y Docencia Económicas, México.

Shorrocks, Anthony (1983), "Ranking income distributions", Economica, 50, Blackwell Publishing, Londres, pp. 3-17.

Tercer Informe Presidencial, Vicente Fox (2003), "Anexo Estadístico", Presidencia de la República, México. 
Recibido: 8 de diciembre de 2008. Aceptado: 2 de diciembre de 2010.

César Octavio Vargas-Téllez. Es doctor en economía aplicada por la Universitat Autónoma de Barcelona. Actualmente es jefe de la División de Estudios Profesionales en la Facultad de Economía de la unAm. Sus líneas de investigación son: pobreza, desigualdad, redistribución de la renta y transferencias públicas, e incidencia fiscal. Entre sus publicaciones destacan: "Purchasing Power Parity across Mexican Cities: A Data Panel Analysis", Applied Economics, 40, Warwick, Londres, pp. 1-9 (2008); "Desigualtat i estat del benestar a Amèrica Llatina", Diàlegs Revista d'Estudis Polítics $i$ Socials, 9 (34), Fundación IneHCA, Barcelona, pp. 93-117 (2006). 